\title{
Plankton bloom controlled by horizontal stirring
}

\author{
W. McKiver ${ }^{1}$, Z. Neufeld ${ }^{1}$, and I. Scheuring ${ }^{2}$ \\ ${ }^{1}$ School of Mathematical Sciences \& Complex and Adaptive Systems Laboratory, University College Dublin, Ireland \\ ${ }^{2}$ Institute of Biology, Department of Plant Taxonomy and Ecology, Research Group of Theoretical Biology and Ecology, \\ Eötvös University, Budapest, Hungary
}

Received: 24 July 2009 - Revised: 22 September 2009 - Accepted: 23 September 2009 - Published: 30 October 2009

\begin{abstract}
Here we show a simple mechanism in which changes in the rate of horizontal stirring by mesoscale ocean eddies can trigger or suppress plankton blooms and can lead to an abrupt change in the average plankton density. We consider a single species phytoplankton model with logistic growth, grazing and a spatially non-uniform carrying capacity. The local dynamics have multiple steady states for some values of the carrying capacity that can lead to localized blooms as fluid moves across the regions with different properties. We show that for this model even small changes in the ratio of biological timescales relative to the flow timescales can greatly enhance or reduce the global plankton productivity. Thus, this may be a possible mechanism in which changes in horizontal mixing can trigger plankton blooms or cause regime shifts in some oceanic regions. Comparison between the spatially distributed model and Lagrangian simulations considering temporal fluctuations along fluid trajectories, demonstrates that small scale transport processes also play an important role in the development of plankton blooms with a significant influence on global biomass.
\end{abstract}

\section{Introduction}

In the open ocean, the dynamics of phytoplankton ecosystems are strongly influenced by the physical characteristics of the environment. The interaction between the physical environment and the ecosystem dynamics can lead to abrupt changes in phytoplankton composition and densities. Phytoplankton bloom (rapid increase of population density, Steele and Henderson, 1992), is frequently observed in phytoplankton communities. While annual cycles of early spring blooms are correlated with the periodic changes in water

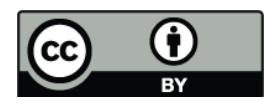

Correspondence to: $\mathrm{W}$. McKiver (william.mckiver@ucd.ie) temperature, light and stratification, there are also many irregular bloom events that do not simply follow the seasonal cycle. Sometimes temporary modifications in the physical environment can cause persistent and abrupt changes in the ecosystem known as regime shifts (Scheffer et al., 2001; deYoung et al., 2008). One typical example of such a regime shift is eutrophication in shallow lakes, where increased phosphorus concentration in the lake may lead to an abrupt rise of some phytoplankton densities, which suppress submerged plants and consequently zooplankton and fish populations (Scheffer, 1990; Scheffer et al., 1993, 2001). Another example of harmful blooms are red tide events, associated with high concentrations of the phytoplankton species Karenia brevis, usually occurring in coastal areas (Yentsch et al., 2008).

From a dynamical point of view, there is a common mechanism for bloom and regime shift: namely, that some external effects cause a change of state within a dynamical system with multiple stable states (Scheffer et al., 2001; Yoshiyama and Nakajima, 2002; Amemiya et al., 2007). The first conceptual models of bloom and regime shifts were based on bi-stable systems of single populations, that have been extended to coupled models of two or more species (Collie et al., 2004), and investigated how changing control parameters of the ecosystem dynamics can trigger jumps between the stable states. If control parameters change slowly, this can generate regime shifts (Scheffer et al., 2001), whereas faster and generally periodic variation of the control parameters can produce blooms in the form of a sudden temporary increase of the population (Stone and Berman, 1993; Truscott, 1995; Franke et al., 1999; Huppert et al., 2002; Huppert et al., 2005).

In addition to temporal changes, the phytoplankton density is typically non-uniform in space, indicating the influence the ocean transport processes have on the dynamics of plankton ecosystems (Martin et al., 2002; Martin, 2003). The effects of small-scale turbulent transport has been studied in

Published by Copernicus Publications on behalf of the European Geosciences Union and the American Geophysical Union. 
the context of vertical mixing (Huisman et al., 2002, 2006) while large scale horizontal flow was shown to determine the statistical properties (e.g. power spectrum) of spatial fluctuations (Abraham, 1998; Hernández-Garcia et al., 2002), and can influence the dynamics of competing species (Károlyi et al., 2000). The role of mixing in the propagation of plankton blooms triggered by localized transient perturbations (e.g. in ocean fertilization experiments) was studied by Abraham et al. (2000) and Neufeld et al. (2002).

Recent work has shown that the stirring rate not only affects the spatial distribution but also has an influence on the total biomass (or average plankton density) when the spatially non-uniform state is maintained by non-uniform input of nutrients or carrying capacity (e.g. due to enhanced vertical transport in some regions) (Pasquero, 2005; Birch et al., 2007; Sandulescu et al., 2007; McKiver and Neufeld, 2009). While in the previous studies, the average plankton concentration was found to be a smooth function of the stirring rate, here we explore the possibility of sudden abrupt changes in a locally bi-stable single population model of phytoplankton dynamics with spatial inhomogeneity of a limiting resource. Both the plankton distribution and the limiting resource are mixed by a 2-D turbulent flow. We show that, for this model, even small changes in the biological timescales relative to the flow timescales can greatly enhance the plankton productivity. Thus, this may be a mechanism by which changes in the rate of horizontal transport can trigger or suppress plankton blooms in the ocean. In Sect. 2 we introduce the model we used and analyze its bi-stable properties. In Sect. 3 we present the results and discuss their implications in Sect. 4.

\section{Model}

\subsection{Coupled fluid-ecosystem model}

To investigate the effects of advection on the total plankton biomass in a spatially non-uniform system with locally bistable population dynamics, we consider a simple wellknown example of a bi-stable predator-prey model with logistic growth and Holling type III functional response and constant predator (i.e. zooplankton) population

$$
\frac{\partial P}{\partial t}+\boldsymbol{u} \cdot \nabla P=r P\left(1-\frac{P}{K(\mathbf{x})}\right)-\frac{Z P^{2}}{S^{2}+P^{2}},
$$

where $P \equiv P(\mathbf{x}, t)$ is the phytoplankton distribution, $r$ is the maximum phytoplankton growth rate, $K(\mathbf{x})$ is the nonuniform carrying capacity, $Z$ is the zooplankton grazing rate and $S$ is the half-saturation constant of the grazing.

In the case of constant carrying capacity, the population density becomes uniform in space and consequently advection does not have any influence on the population dynamics. However, the availability of nutrients and other external conditions typically lead to spatial variability in the phytoplankton density. To represent this, we consider a spatially non- uniform carrying capacity $K(\mathbf{x}) \equiv K_{0}+\delta \cos (x+y)$, which is characterized by the mean $K_{0}$ and the amplitude of the variability $\delta$. For simplicity, we assume that the carrying capacity is fixed in space and time-independent. Although, for real systems the carrying capacity can be time-dependent, since the fluid elements are advected by the flow across different regions, this simplification does not affect the qualitative behaviour of the system.

The two-dimensional velocity field $\boldsymbol{u}(\mathbf{x}, t)=(u, v)$ represents a typical mesoscale ocean flow on length-scales of the order of a few hundred kilometers. The flow field is obtained by solving the 2-D incompressible Navier-Stokes equations with forcing and dissipation (Tabeling, 2002), namely

$$
\frac{\partial \zeta}{\partial t}+\boldsymbol{u} \cdot \nabla \zeta=F+D
$$

where $\zeta \equiv \partial v / \partial x-\partial u / \partial y$ is the vorticity field which is a scalar in 2-D flows, $D$ is the dissipation and $F$ is the forcing. The dissipation is a combination of hyperviscosity of the form $D_{h i}=-v \nabla^{8} \zeta$ which acts at small scales, and linear friction given by $D_{l o}=-\alpha \zeta$, that prevents accumulation of energy at the largest scales via the inverse cascade (see Maltrud and Vallis, 1991). The forcing is applied in spectral space at the large scale wavenumber of $k_{f}=10$, and has a fixed amplitude but with a random phase. This forcing inputs energy at large scales which is relevant to the type of large scale forcing that exists in the ocean.

As was done previously (McKiver and Neufeld, 2009), the plankton model can be characterised in terms of the parameter $\gamma \equiv T_{B} / T_{F}$ where $T_{B}$ is the biological timescale and $T_{F}$ is the flow timescale. We define the biological timescale as the inverse of the growth rate, i.e. $T_{B} \equiv 1 / r$, and the flow timescale as $T_{F}=L / U$, where $L$ is the forcing lengthscale $L \equiv 2 \pi / k_{f}$ and $U$ is the root-mean-square velocity field. Thus $\gamma$ is given by

$\gamma=\frac{U}{r L}$

and Eq. (1) can then be written in dimensionless form as

$$
\frac{\partial P}{\partial t}+\boldsymbol{u} \cdot \nabla P=\frac{1}{\gamma}\left[P\left(1-\frac{P}{K(\mathbf{x})}\right)-\frac{\beta P^{2}}{1+P^{2}}\right]
$$

where the plankton concentrations and carrying capacity are measured in units of $S$, and the parameter $\beta=Z / r$ is a ratio of the grazing rate to the growth rate. Here we fix the characteristic fluid timescale $T_{F}$ to unity so that our time units are defined in units of $T_{F}$, but we vary the timescale ratio $\gamma$ by varying the growth rate, $r$.

\subsection{Numerical methods}

The ecosystem Eq. (4) is solved on a doubly periodic square domain of size $2 \pi$ using a semi-Lagrangian scheme with a 
grid resolution of 1024 squared. Since typically the forcing scale in the ocean is of the order of $50 \mathrm{~km}$ (Bracco et al., 2000) and our forcing scale is $k_{f}=10$, then our domain size corresponds to $500 \mathrm{~km}$ with a grid resolution of about $0.5 \mathrm{~km}$. The advecting fluid velocity is obtained by solving Eq. (2) using the pseudo-spectral method. A fourth order Runge-Kutta scheme is used for the time integration of both equations with a time step of $d t=4 \times 10^{-3} T_{F}$ which is less than half the CFL time step required for stability. In the semiLagrangian method, the plankton dynamics is solved within fluid parcels, whose motion are tracked using the midpoint method and then calculated on grid points using bicubic interpolation (see Bartello and Thomas, 1996; Temperton and Staniforth, 1987 for details of the semi-Lagrangian method). In our model, we do not add diffusion explicitly, however some numerical diffusion is always present as a result of using interpolation in the semi-Lagrangian scheme (Dritschel et al., 1999). This numerical diffusion can be estimated using $D_{n} \approx d x^{2} / d t$ (see Sandulescu et al., 2007, for details). To examine if this diffusion has any effect on the ecosystem dynamics, we also use a non-diffusive fully-Lagrangian model to solve the ecosystem Eq. (4). This Lagrangian model uses the flow field, which is still obtained by solving the NavierStokes equations on the grid, to advect a plankton particle along a single long trajectory of a fluid element in the turbulent flow (McKiver and Neufeld, 2009).

\subsection{Bi-stable solutions of the local dynamics}

When the carrying capacity is uniform in space $(\delta=0)$ and there is no fluid advection the (nontrivial) equilibria for this system is given by the solution of

$P_{E}^{3}-K P_{E}^{2}+(1+\beta K) P_{E}-K=0$

where $P_{E}$ is the equilibrium plankton concentration. This equation has three real solutions when

$-4 a^{2}+\left(b^{2}+18 b-27\right) a-4 b^{3}>0$

where $a \equiv K^{2}$ and $b \equiv 1+\beta K$. When this condition is satisfied, the system is bi-stable. In Fig. 1a we show the region for which the system is bistable.

A previous study has shown that in the presence of advection, the global carrying capacity of the system is affected by how fast the flow is in relation to the ecosystem timescales (see McKiver and Neufeld, 2009). In essence, there is an effective carrying capacity, $K_{\text {eff }}$, which depends on the timescale ratio $\gamma=T_{B} / T_{F}$. In McKiver and Neufeld (2009) it was shown that without grazing $(\beta=0)$ the value of $K_{\text {eff }}$ decreases monotonically with $\gamma$, from the average carrying capacity $\langle K\rangle$ to the harmonic mean $1 /\langle 1 / K\rangle$ (where $\langle$. $\rangle$ indicate spatial average). For Eq. (4) the existence of the bistable states depends on the effective carrying capacity $K_{\text {eff }}$. If $\langle K\rangle$ is within the bi-stable regime, but the harmonic mean $1 /\langle 1 / K\rangle$ is outside of this bi-stable regime, we can expect (a)
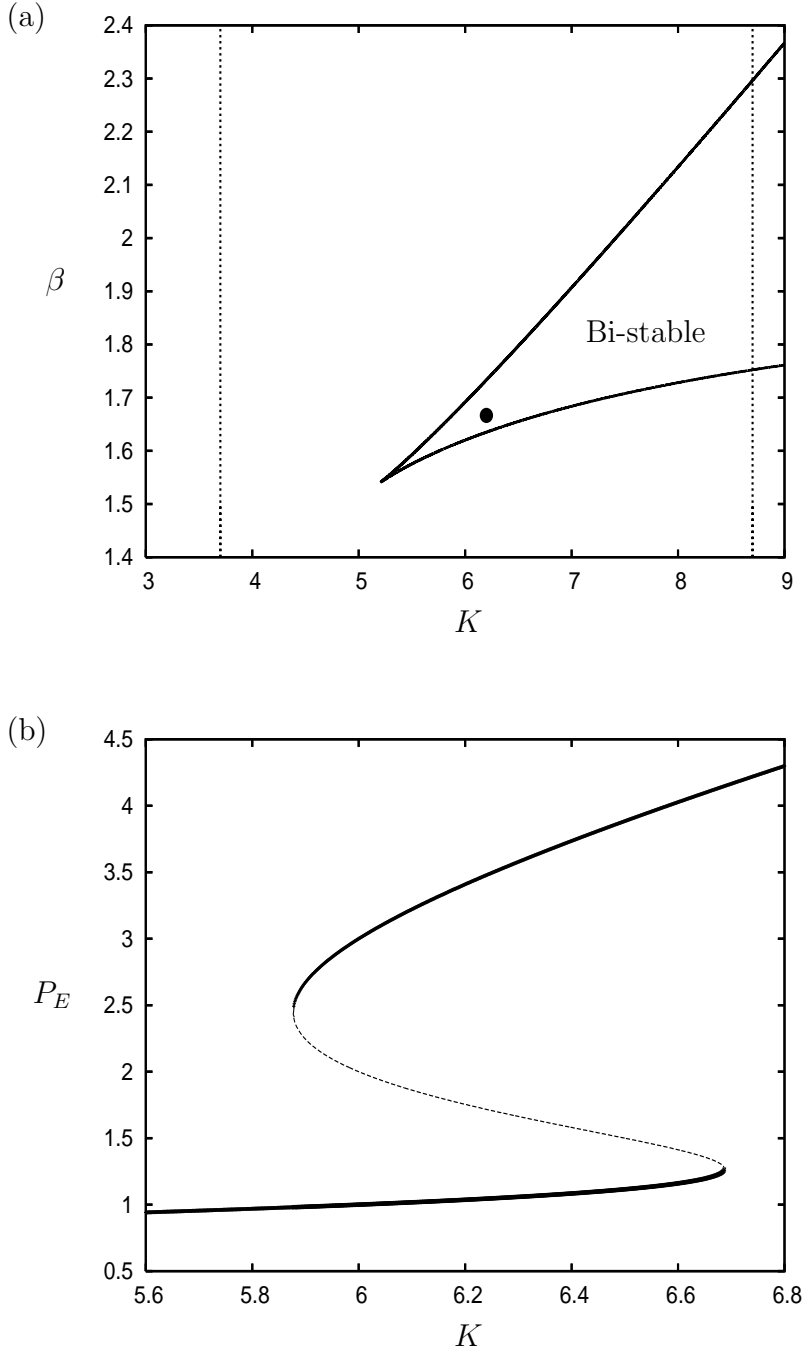

Fig. 1. (a) Plot indicating parameter range over which the system is bi-stable. The point indicates the parameter values used $K_{0}=6.2$ and $\beta=1.67$, whereas the dashed lines indicates the minimum and maximum carrying capacity values $\left(K_{0} \pm \delta\right)$. (b) Plot showing how the equilibrium solution $P_{E}$ depend on $K$ for $\beta=1.67$. The solid curves are the stable solutions and the dashed line is the unstable solution.

that a transition will occur as the value of $\gamma$ is changed, in other words decreasing the rate of flow can trigger a plankton bloom.

To illustrate this effect, we choose the parameter values $\beta=1.67, K_{0}=6.2$ and $\delta=2.5$. For these parameter values, when $K_{\text {eff }}=K_{0}$ the system is locally bi-stable with stable equilibrium values of 1.0 and 3.4. Due to the spatially nonuniform carrying capacity, in the absence of mixing the system would be composed of patches of bi-stable local dynamics and regions where the local dynamics is mono-stable corresponding to the upper or lower solution branches shown in Fig. 1b. In the presence of mixing, the fluid parcels move 
across the different types of locally bi-stable and mono-stable regions and, therefore, the rate at which these changes occur can have a non-trivial effect on the global ecosystem dynamics. The limitation of very fast mixing when the system is almost uniform in space, the global behaviour is described by the harmonic mean carrying capacity that for the above parameters is $1 /\langle 1 / K\rangle=5.67$, which in our example falls outside of the bi-stable regime which ranges from $5.87 \leq K \leq 6.68$ for $\beta=1.67$ (see Fig. 1b).

\section{Results}

We perform a number of simulations for a large range of values of the parameter $\gamma$. We integrate the equations until the mean plankton has reached a statistically stationary steady state. The time taken to reach this steady state increases with $\gamma$ as was found in McKiver and Neufeld (2009). Typically for mesoscale flows $\gamma$ is less than unity, for example if $r=0.5 d^{-1}, U=1 \mathrm{cms}^{-1}$ and $L=50 \mathrm{~km}$, using Eq. (3) we get $\gamma \approx 0.34$. However, $\gamma$ can vary depending on the region of ocean being considered and also depending on the seasons which affect the phytoplankton growth rate.

In Fig. 2, we show plots of the plankton field after the steady-state is reached. As $\gamma$ increases the solution goes from being composed of patches of high and low concentration (for $\gamma \lesssim 0.6$ ), to the mono-stable regime where only low concentration values exist (for $\gamma \gtrsim 0.6$ ). For the low $\gamma$ values $(\leq 0.02)$ the spatial structure of the plankton field closely follows the equilibrium value corresponding to the local carrying capacity field. In the bi-stable regions in space where there are multiple equilibria, the plankton concentration is determined by whether the fluid trajectory is coming from a low or a high carrying capacity region corresponding to the lower and upper solution branches. Thus, when there is any arbitrarily slow mixing, this eliminates the co-existence of multiple steady-states in the spatially distributed system, and so there is a unique solution which is independent of the initial condition. Nevertheless, a consequence of the bistable dynamics remains the sharp transition in space separating bloom and low concentration regions.

As $\gamma$ increases more structures characteristic of fluid advection can be seen within the plankton concentration field. The advective transport process becomes more important as fluid parcels move faster from regions of different properties, therefore, the population dynamics cannot reach the local equilibrium. When $\gamma$ is increased further, the spatial distribution becomes more uniform in space. While the transition from a plankton field dominated by the spatial distribution imposed by the external parameters to a uniform system is similar to that seen in the case without grazing (McKiver and Neufeld, 2009), an important difference in the present model is that here the average concentration changes abruptly with $\gamma$ (Fig. 3). This is a consequence of the fact that increased mixing reduces the effective carrying capacity that suppresses the high concentration branch as the average behaviour of the system moves outside of the bi-stable regime and the solution branch having low plankton concentration dominates everywhere in space.

Note, that although the local dynamics has multiple steady-states, the spatial model with mixing has a unique spatially distributed solution for any value of the parameter $\gamma$. Consequently, there is no hysteresis associated with this kind of regime shift, i.e. the abrupt change in the systems behaviour takes place at the same value regardless whether the transition is reached from below or above by increasing or reducing the timescale ratio $\gamma$.

In order to illustrate the mechanism of the transition, we consider a simple model of the population dynamics where the advection is replaced by a time-dependent carrying capacity, i.e.

$$
\frac{d P}{d t}=\frac{1}{\gamma}\left[P\left(1-\frac{P}{K_{0}+\delta \cos (\omega t)}\right)-\frac{\beta P^{2}}{1+P^{2}}\right]
$$

where $P(t)$ represents the time-dependent phytoplankton concentration in a moving fluid parcel, and the carrying capacity changes with frequency $\omega$. The frequency $\omega$ corresponds to the timescale, $T_{C}=2 \pi / \omega$, which is the period of oscillation of the carrying capacity and represents the characteristic timescale for advected fluid elements to move between regions of low and high carrying capacity. In our model, this timescale is different than the flow timescale $T_{F}$, since the typical vortices are smaller than the bands of the carrying capacity field and, hence, typically takes longer for a fluid particle to move from different regions of carrying capacity. In McKiver and Neufeld (2009) the timescale $T_{C}$ was determined for this flow by examining the decay of the correlation of the carrying capacity measured along an ensemble of Lagrangian trajectories and they found the characteristic correlation time to be $T_{C} \approx 8 T_{F}$. Thus, we can estimate the timescale ratio $\gamma \equiv T_{B} / T_{F}$ where we use the same biological timescale, $T_{B}=1 / r$, but the flow timescales $T_{F}=T_{C} / 8$. This system can be easily solved for different values of $\gamma$.

In Fig. 3, we plot the equilibrium mean and variance of plankton as a function of $\gamma$ obtained by solving the full Eqs. (4) using both the grid based semi-Lagrangian method (dash-dots) and a fully-Lagrangian method (crosses), as well as the solution obtained from the simpler time-dependent carrying capacity model (Eq. 7) (solid curve). Clearly the transition from the bi-stable regime can be seen to occur when $\gamma \approx 0.6$. However, for the simpler time-varying carrying capacity model there is no jump but rather a smooth transition to the lower branch. This is because of the absence of diffusion in the simplified model, since this in the semiLagrangian model can lead to the advective propagation of the localized bloom events as it was shown in Neufeld et al., 2002. This is demonstrated by the solution obtained using the non-diffusive Lagrangian method (crosses), which also show a smooth transition to the lower branch, consistent with 


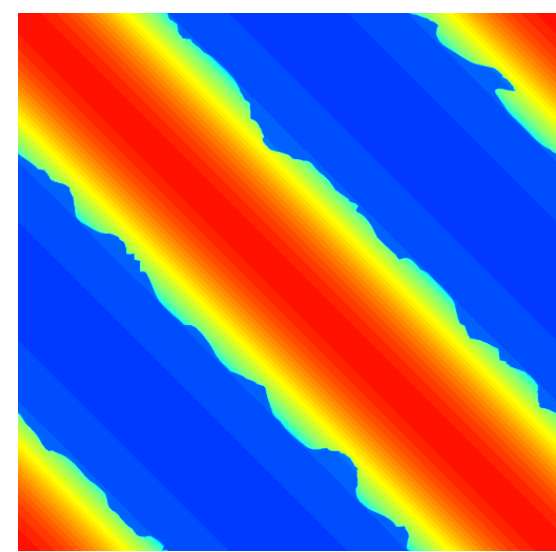

(a) $\gamma=0.0002$

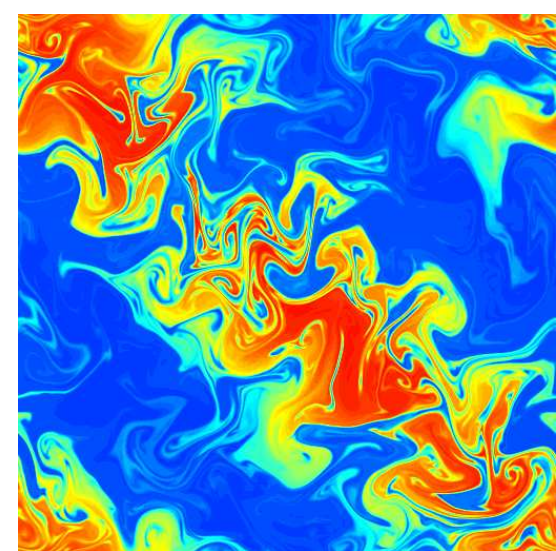

(c) $\gamma=0.2$

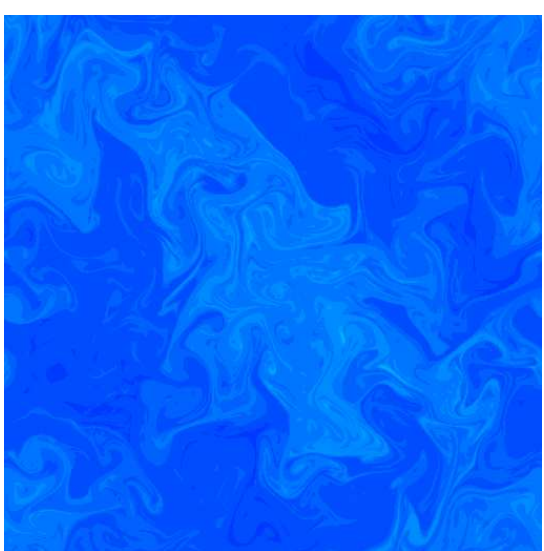

(e) $\gamma=0.7$

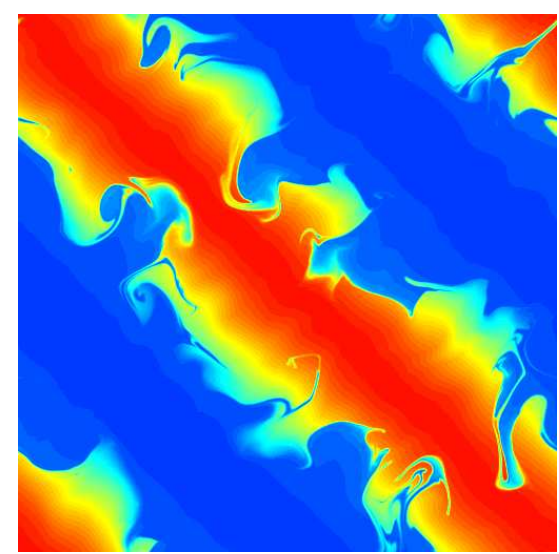

(b) $\gamma=0.02$

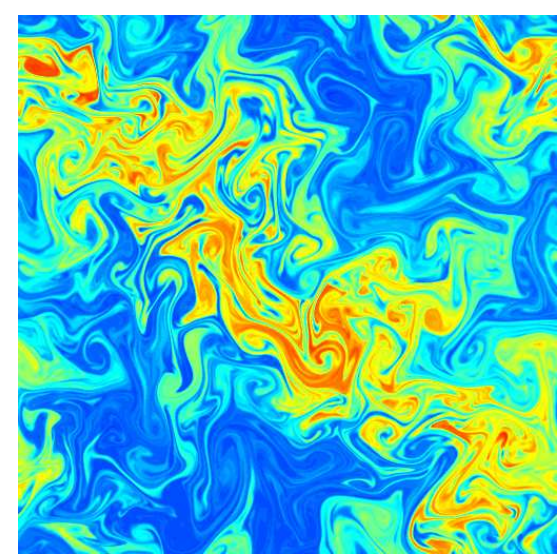

(d) $\gamma=0.6$

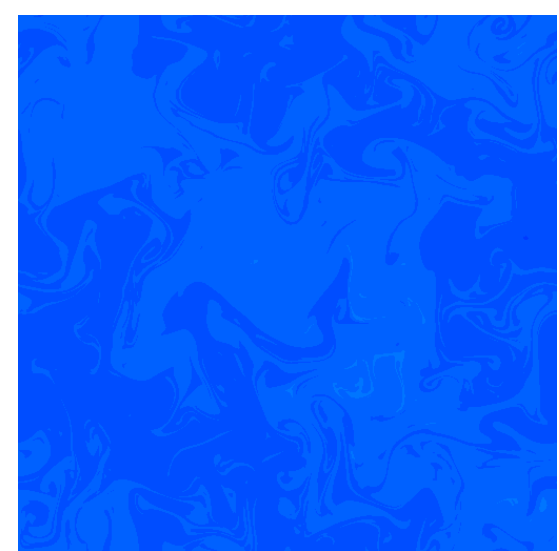

(f) $\gamma=1.8$

Fig. 2. Snapshots of the plankton field (after statistically stationary state is reached) for $\gamma=0.0002,0.02,0.2,0.6,0.7$ and 1.8 (going from left to right and from top to bottom). The minimum and the maximum contour values are 0.54 (blue) and 6.68 (red).

the simpler time-varying carrying capacity model. Thus, this simple model does give a qualitative estimate of the $\gamma$ value where the jump occurs, as can be seen where the variance goes to zero, and also shows that the sharp transition in the global biomass is due to the small scale turbulent diffusion.

\section{Conclusions}

Phytoplankton blooms are created by the coexistence of many complex factors. We have demonstrated that the dynamics of phytoplankton blooms can be affected by 
(a)

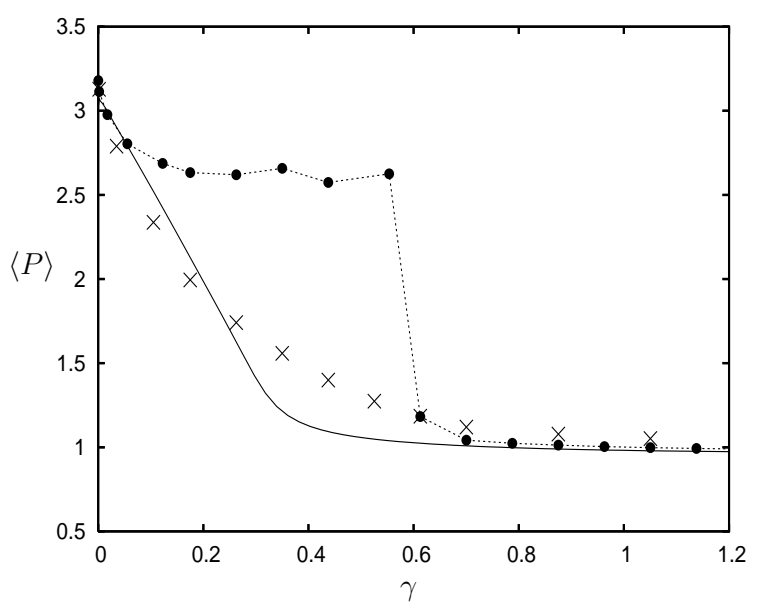

(b)

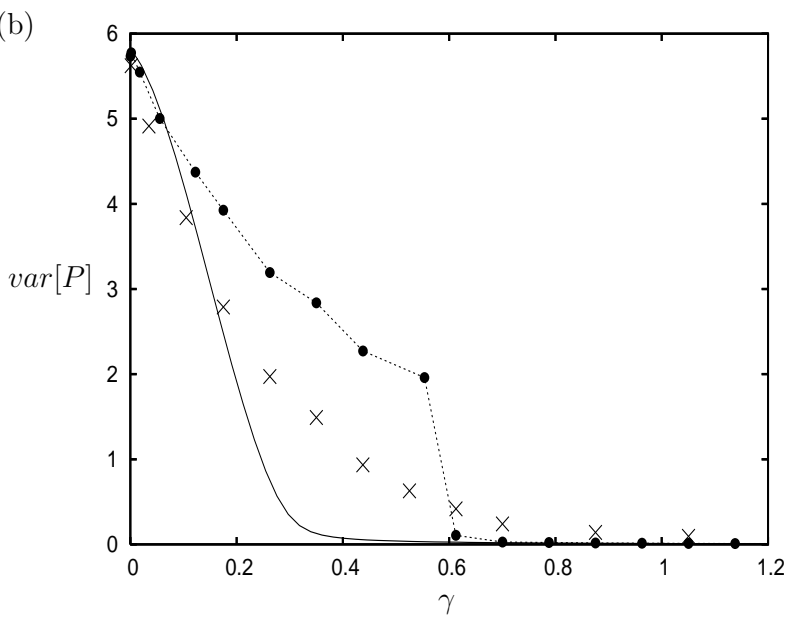

Fig. 3. The equilibria of (a) mean and (b) variance of plankton versus $\gamma$ using the full model with diffusion (dash-dots), without diffusion (crosses) and the simple time-dependent carrying capacity (solid curve).

horizontal stirring in a turbulent flow and by the bi-stability properties of the biological system. We show that the existence of a bloom is sensitive to the ratio of biological and hydrodynamical time scales $(\gamma)$, thus, highlighting the importance of horizontal ocean advection in the generation of plankton blooms. When the flow stirring rate is small relative to the growth rate of phytoplankton the system is within the bi-stable regime, where both high and low carrying capacity values exist, and hence there are blooms. However, beyond a certain flow stirring rate (relative to the phytoplankton growth rate) the system undergoes a transition to the single equilibrium case, with a uniform low plankton concentration. One possible example of this in the real ocean could be when increased sunlight with stratification temporally increases the growth rate of phytoplankton ( $T_{B}$ decreases), while stratification generally is associated with a decreased level of hor- izontal mixing ( $T_{F}$ increases) (Miller, 2004). Consequently, increased sunlight and stratification could lead to smaller $\gamma$ values, that increases the probability of a bloom associated with a sudden shift to a higher average biomass state.

Recently Rossi et al. (2008) studied the relation between the mixing and phytoplankton concentration of the Benguela and Canary upwelling systems. They measured the monthly average of the finite size Lyapunov exponents (FSLE) of horizontal mixing, which estimates the time scale of the mixing as $\left(\mathrm{FSLE} \propto 1 / T_{F}\right)$. Both upwelling systems are divided into two regions according to mixing activity. They found very different chlorophyll concentrations in the different regions. In accordance with the predictions of our simple model, for both systems the subregions with more mixing activity (lower $T_{F}$ ) were found to have lower chlorophyll concentrations.

While the large change in the global biomass in the present model is primarily caused by the interplay between horizontal advection and the locally bi-stable population dynamics within a spatially non-uniform environment, we have shown that small-scale diffusive transport (representing turbulent mixing on typical lengthscales of a few tens of meters in the real ocean) is responsible for the sharp transition in the total plankton concentration. This suggests, that a quantitatively correct representation of these sub-grid scale transport processes is crucially important in large scale ocean-ecosystem models, as has been found in other previous studies (see for example Lévy et al., 2001).

Here we have assumed the carrying capacity is time independent. Though this is a simplification of the system, a time independent carrying capacity, while changing the spatial structure of the phytoplankton field (particularly at small $\gamma$ ) would not change the qualitative features of the sharp transition in the biomass as a function of $\gamma$, as this is dependent on the effective carrying capacity, which varies between $\langle K\rangle$ and $1 /\langle 1 / K\rangle$ (McKiver and Neufeld, 2009). Qualitatively similar behaviour is also expected in the case when the carrying capacity is described explicitly as a field advected by the flow (see Pasquero et al., 2005, for an example of a model where the nutrient field is included explicitly as a variable) as long as its non-uniformity is maintained by a spatially distributed source and assuming that it is independent from the phytoplankton distribution (i.e. the feed-back due to nutrient uptake is negligible).

Overall, this work demonstrates the important effect horizontal advection can have on phytoplankton biomass using a simple idealized model. However, the effect of a dynamical zooplankton species is neglected here and further study is needed to see if the strong dependence of biomass on horizontal advection is seen in a more realistic ecosystem model. Multiple steady states have been found in more complicated ecosystem models (Edwards and Yool, 2000) and we expect similar stirring induced transitions can take place in complex ecosystem models too. 
Acknowledgements. W. M. and Z. N. have obtained support for this research from the Irish Research Council for Science, Engineering and Technology (IRCSET) and Science Foundation Ireland (SFI) RFP research grant, while I. S. is supported by the Hungarian Science Foundation (OTKA) under grant T72037. Also we wish to acknowledge the SFI/HEA Irish Centre for High-End Computing (ICHEC) for the provision of computational facilities and support.

Edited by: C. López

Reviewed by: two anonymous referees

\section{References}

Abraham, E. R.: The generation of plankton patchiness by turbulent stirring, Nature, 391, 577-580, 1998.

Abraham, E. R., Law, C. S., Boyd, P. W., Lavender, S. J., Maldonado, M. T., and Bowie, A. R.: Importance of stirring in the development of an iron-fertilized phytoplankton bloom. Nature, 407, 727-730, 2000.

Amemiya, T., Enomoto, T., Rossberg, A. G., Yamamoto, T., Inamori, Y., and Kiminori, I.: Stability and dynamical behavior in a lake-model and implications for regime shifts in real lakes Ecological Modelling, 206, 54-62, 2008.

Bartello, P. and Thomas, S.: The cost-effectiveness of semiLagrangian advection, Mon. Weather Rev., 124, 2883-2897, 1996.

Birch, D. A., Tsang, Y. K., and Young, W. R.: Bounding biomass in the Fisher equation, Phys. Rev. E, 75, 066304, doi:10.1103/PhysRevE.75.066304, 2007.

Bracco, A., Provenzale, A., and Scheuring, I.: Mesoscale vortices and the paradox of the plankton, Proc. R. Soc. Lond. B, 267, 1795-1800, 2000.

Collie, J. S., Richardson, K., and Steele, J. H.: Regime shifts: can ecological theory illuminate the mechanisms?, Prog. Oceanogr., 60, 281-302, 2004.

deYoung, B., Barange, M., Beaugrand, G., Harris, R., Perry, R. I., Scheffer, M., and Werner, F.: Regime shifts in marine ecosystems: detection, prediction and management, Trends Ecol. Evol., 23, 402-409, 2008.

Dritschel, D., Polvani, L., and Mohebalhojeh, A.: The contouradvective semi-Lagrangian algorithm for the shallow water equations, Mon. Weather Rev., 127, 1551-1565, 1999.

Edwards, A. M. and Yool, A.: The role of higher predation in plankton population models, J. Plank. Res., 22, 1085-1112, 2000.

Franke, U., Hutter, K., and John, K.: A physical-biological coupled model for algal dynamics in lakes, B. Math. Biol., 61, 239-272, 1999.

Hernández-García, E., López, C., and Neufeld, Z.: Small-scale structure of nonlinearly interacting species advected by chaotic flows, Chaos, 12(2), 470-480, 2002.

Huisman, J., Arrayás M., Ebert, U., and Sommeijer, B.: How do sinking phytoplankton species manage to persist?, American Naturalist, 159, 245-254, 2002.

Huisman, J., Nga, N., Thi, P., Karl, D. M., and Sommeijer, B.: Reduced mixing generates oscillations and chaos in the oceanic deep chlorophil maximum, Nature, 439, 322-325, 2006.

Huppert, A., Blasius, B., and Stone, L.: A model of phytoplankton blooms. American Naturalist, 159, 156-171, 2002.
Huppert, A., Blasius, B., Olinky, R., and Stone, L.: A model for seasonal phytoplankton blooms, J. Theor. Biol., 236, 276-290, 2005.

Károlyi, G., Péntek, Á., Scheuring, I., Tél, T., and Toroczkai, Z.: Chaotic flow: the physics of species coexistence, Proc. Natl. Acad. Sci. USA, 97(25), 13661-13665, 2000.

Lévy, M., Klein, P., and Treguier, A.: Impact of sub-mesoscale physics on production and subduction of phytoplankton in an oligotrophic regime, J. Mar. Res., 59, 535-565, 2001.

Maltrud, M. E. and Vallis, G. K.: Energy spectra and coherent structures in forced two-dimensional and beta-plane turbulence, J. Fluid Mech., 228, 321-342, 1991.

Martin, A. P., Richards, K., Bracco, A., and Provenzale, A.: Patchy productivity in the open ocean. Global Biogeochem. Cycles, 16, 2, doi:10.1029/2001GB001449, 2002.

Martin, A.: Phytoplankton patchiness: the role of lateral stirring and mixing, Prog. Oceanogr., 57, 125-174, 2003.

McKiver, W. and Neufeld, Z.: The influence of turbulent advection on the statistical properties of plankton ecosystems, Phys. Rev. E, 79, 061902, doi:10.1103/PhysRevE.79.061902, 2009.

Miller, C.: Biological Oceanography, Blackwell Publ., UK, 2004.

Neufeld, Z., Haynes, P., Garçon, V., and Sudre, J.: Ocean fertilization experiments may initiate a large scale phytoplankton bloom, Geophys. Res. Lett., 29(11), 1534, doi:10.1029/2001GL013677, 2002.

Pasquero, C.: Differential eddy diffusion of biogeochemical tracers, Geophys. Res. Lett., 32, L17603, doi:10.1029/2005GL023662, 2005.

Pasquero, C., Bracco, A., and Provenzale, A.: Impact of spatio-temporal variability of the nutrient flux on primary productivity in the ocean, J. Geophys. Res., 110, C07005, doi:10.1029/2004JC002738, 2005.

Rossi, V., López, C., Sudre, J., Hernández-García, E., and Garçon, V.: Comparative study of mixing and biological activity of the Benguela and Canary upwelling systems, Geophys. Res. Lett., 35, L11602, doi:10.1029/2008GL033610, 2008.

Sandulescu, M., López, C., Hernández-García, E., and Feudel, U.: Plankton blooms in vortices: the role of biological and hydrodynamic timescales, Nonlin. Processes Geophys., 14, 443-454, 2007 , http://www.nonlin-processes-geophys.net/14/443/2007/.

Scheffer, M.: Multiplicity of stable states in freshwater systems, Hydrobiologia, 200/201, 475-486, 1990.

Scheffer, M., Hosper, S. H., Meijer, M. L., and Moss, B.: Alternative equilibria in shallow lakes, Trends. Ecol. Evol., 8, 275-279, 1993.

Scheffer, M., Carpenter, S., Folley, J. A., Folke, C., and Walker, B.: Catastrophic shifts in ecosystems, Nature, 413, 591-596, 2001.

Steele, J. H. and Henderson, E. W.: The role of predation in plankton models, J. Plank. Res., 14, 157-172, 1992.

Stone, L. and Berman, T.: Positive feedback in aquatic ecosystems: the case of microbial loop, B. Math. Biol., 55, 919-936, 1993.

Tabeling, P.: Two-dimensional turbulence: a physicist approach, Phys. Reports, 362, 1-62, 2002.

Temperton, C. and Staniforth, A.: An efficient two-time-level semiLagrangian semi implicit integrating scheme, Q. J. Roy. Meteorol. Soc., 113, 1025-1040, 1987.

Truscott, J. E.: Environmental forcing of simple plankton models, J. Plank. Res., 17, 2207-2232, 1995. 
Yentsch, C. S., Lapointe, B. E., Poulton, N., and Phinney, D. A.: Anatomy of a red tide bloom off the southwest coast of Florida, Harmful Algae, 7(6), 817-826, 2008.
Yoshiyama, K. and Nakajima, H.: Catastrophic Transition in Vertical Distributions of Phytoplankton: Alternative Equilibria in a Water Column, J. Theor. Biol., 216, 397-408, 2002. 